\title{
Comparative Analysis of Object-Based and Pixel-Based Classification of High-Resolution Remote Sensing Images for Mapping Coral Reef Geomorphic Zones
}

\author{
Muhammad Lutfi Mahasinul Akhlaq ${ }^{1 *}$, Gatot Winarso ${ }^{2}$
}

\author{
${ }^{1}$ Informatic Management Department, STMIK Bina Patria, Magelang, Indonesia \\ ${ }^{2}$ Remote Sensing Application Centre, LAPAN, Jakarta, Indonesia \\ *Corresponding author. Email: lutfi@stmikbinapatria.ac.id
}

\begin{abstract}
Coral reefs ecosystem has great value in terms of economy, culture, and biology for the global society and is the most productive and diverse biological ecosystem in the world. Earth observation from space so called remote sensing technology by using high-resolution Satellite, offers powerful capabilities for understanding, forecasting, managing, monitoring and decision making about coral reefs ecosystem. This study focuses on the comparison analysis between Object Base and Pixel Base image classifications of remote sensing imagery for Mapping Coral Reef Geomorphic Zones in the Karimunjawa National Park. The accuracy of each method was assessed using reference data sets derived from high-resolution satellite images, aerial photograph and field investigation. The accuracy of geomorphology zone mapping used Object Base Classification technique indicates that the overall accuracy (OA) was $88,62 \%$. while the pixel-based classification produces the low overall accuracy was $73 \%$. This research suggest that the object-based technique could be a promise approach for mapping coral reef geomorphic zones, where the information obtained from this research was more accurate. In paper, we observed that the object-based technique shows higher accuracy in classification process than the pixel-based technique because pixel based can't satisfy the high-resolution satellite data properties and it produced data redundancy.
\end{abstract}

Keywords: image processing, image, remote sensing

\section{INTRODUCTION}

Coral reefs ecosystem has great value in terms of economy, culture, and biology for the global society and is the most productive and diverse biological ecosystem in the world [1]. Ocean and land observation from space so called remote sensing technology by using high-resolution satellite, provides powerful capabilities for decision making because satellite captures some data for understanding, forecasting, managing, monitoring and about coral reefs ecosystem.

Accurate, reliable and up-to-date coral reef mapping has significant role for economic issues, food policy and environmental implementation. Since climate changes, human activities and water quality changes need to be monitoring. Compared to conventional methods of surveying, there are many advantages in using remote sensing technology for coral reef mapping. Remote sensing technology is considered as a powerful and useful tool that enables feasible and practical data acquisition to determine either the extent or the geographical distribution of coral reef. Typically, the classification is one of the widely used approaches for the extraction of coral reef geomorphic zones information from remotely sensed data.

IKONOS, Worldview and Quick bird are commercial satellites imagery which are the widespread public availability from space, it was very important development remote sensing technology in the 2000s. The spectral resolution of IKONOS, Worldview and Quick bird was roughly equivalent to Landsat but the spatial resolution was much improved. Many research by remote sensing scientists into the utility of these spatial data for mapping reef communities produced mixed results [2],[3],[4]. Some studies of coral reef geomorphology are based on data fusion by integrating field and remote sensed datasets [5]. High-resolution satellite imagery can be interpreted in some methods. Field observation is common for photographs Visual inspection, while manipulations of digital data and image processing make use of computers. Classification is the most common image analysis step which can enhance the image to improve its visual interpretability and perform the image through a computer image processing system. The reef scientist can get digital image data from classification process and use it as input for further relevant analyses.

Pixel based unsupervised and supervised classification approaches are traditional analyzed methods which are limited in the image properties which they assess data from remote sensing [6]. Object Base Image Analysis (OBIA) is a new popular method in the field of image processing. Papers by [6],[7],[8], [9] all of them use OBIA methods. The reliability of spatial products is very much determined by the level of error in the digital map product. Therefore, understanding, finding and measuring the source of errors related to and contained in digital maps is very important. Validation processes, resulting accuracy measures and their relative remote sensing image products need to understand 
the commonly used as a result for scientists, technicians, and managers [2],[10],[11].

However, the validation process is measuring the difference between the results of image processing in the form of image pixel values and visual references values in the field with the same location and time. This study examines the application of methods with a pixel-based and object-based approach for mapping the geomorphological classification of coral reefs in Karimunjawa by assessing the accuracy of the classification for each method approach through an error matrix.

\section{METHODS}

\subsection{Research Methodology}

The research methodology used is an experimental method with an empirical approach. Field data is used as part of the input in the initial processing and as comparative data between the results of image interpretation with actual field conditions called validation

\subsection{Research Stages}

Table 1 The Rule Set of OBIA Process

\begin{tabular}{|clcc|}
\hline Rule Set & \multicolumn{1}{c|}{ Band } & Algorithm & Classification \\
\hline Rule Set 1 & NIR, GREEN & NIR/GREEN & land : 1-10, water $<1$ \\
\hline Rule Set 2 & Coastal Blue, Green. & Coastal Blue/ Green & Shallow water $<1$ \\
\hline Rule Set 3 & Blue, Green, Red & & Geomorphic Zones \\
\hline
\end{tabular}

Table 2 Coral Reef Geomorphic Zones Segmentation

\begin{tabular}{|llll|}
\hline Object & \multicolumn{1}{c}{ Band } & \multicolumn{1}{c|}{ Algorithm } & \multicolumn{1}{c}{ Classification } \\
\hline lagoon & Blue, Red & Blue/Red $\geq 2.7$ & $\begin{array}{l}\text { Relation Border to lagoon }>0.9 \text { become lagoon; Lagoon with } \\
\text { area }<1000 \text { px become unclassified. }\end{array}$ \\
\hline reef crest & Blue, Red & Blue/Red $\geq 1,6$ & Border to Reef slope $>0$ \\
\hline reef flat & Blue, Red & Unclassified at Geomorphic: Reef Flat \\
\hline reef slope & Blue, Green, Red & Red*Blue/Green $<300$ & $\begin{array}{l}\text { Relations to neighbor object are Border to Deep water and } \\
\text { Border to Reef slope }\end{array}$ \\
\hline
\end{tabular}

Accuracy Assessment, Accuracy comparisons between pixel-based classifications result and object-based classifications result of World View II images are performed using 250 random reference points. producer accuracy, user accuracy, and kappa statistics are used to compile an error matrix derived from index values

\section{RESULTS AND DISCUSSION}

Classifying remote sensing imagery from World View II has been used as a material for pixel and object-based image analysis approaches. Error matrices using the same test area and the same time as reference data have been used in assessing the accuracy of the classification using pixel based and object-based approaches. The results of the accuracy assessment indicate that the object-based analysis
Image Processing. The study was conducted in the Karimunjawa Marine National Park, Jepara, Central Java and was conducted in March-April 2018. World View-2 imagery was acquired in August 2017 (standard level 2A 16 bit) with the UTM Zone 48S-WGS84 coordinate projection system.

Image Characteristics Citra World View-2 consists of 8 multispectral channels (coastal, blue, green, yellow, red, red edge, NIR-1, NIR-2) with spatial resolution of 2 meters and panchromatic with a spatial resolution of 0.5 meters [12]. In this study, supervised classification and object-based image analysis (OBIA) methods were conducted to highresolution multispectral World View imagery to extract information about coral reef geomorphic zones in selected region. Pixel based classification method is a classic method where the classification of remote sensing images is done by pixel per pixel and based on the spectral value of each pixel in the image so that one pixel only belongs to one class. Geomorphological zone classifications are determined from spectral patterns through training samples. In this paper, mapping of coral reefs is based on the geomorphology of coral reefs according to Maxwell [13]. Recognition Software classifies pixels into detailed class hierarchies with membership values for each class and pixels are not only assigned to one class. Fuzzy classification concept classifies an image object has memberships in more than one class. The best classification results are taken from the classification with the highest assignment value approach has a higher producer accuracy for most coral reef geomorphological zone classifications.

Pixel based classification results: Geomorphic zone classification is assessed by comparing the pixel value of images numerically through training samples. The supervised classification process of World View II involves 3 basic stages: the training stage, the classification stage and the accuracy assessment stage.

The accuracy of the classification results is very important, so the accuracy assessment is very essential. Accuracy assessment is done by comparing the results of classification with real objects in the field which are then used to compile the error matrix. Table 1 and Table 2 shows an accuracy assessment result. As we see in Table 3 the overall accuracy for pixel base image classification is $73 \%$. Information classes of "reef crest" has high producer's and user's accuracy, but the information classes of "reef slope" 
has a low producer's and user's accuracy. There is a similarity spectral reflectance between the reef slope, reef crest and reef flat cause low accuracy, this could be due to the miss-classification between these five classes.

Table 3 Accuracy assessment results of Pixel base Classification

\begin{tabular}{|lcccccc|}
\hline ImagelSurvey & Reef Flat & Reef Crest & Reef Slope & Lagoon & Total & UA \\
\hline Reef Flat & 18 & 2 & 2 & 3 & 25 & $72,00 \%$ \\
\hline Reef Crest & 2 & 19 & 3 & 1 & 25 & $76,00 \%$ \\
\hline Reef Slope & 3 & 2 & 18 & 2 & 25 & $72,00 \%$ \\
\hline Lagoon & 2 & 1 & 4 & 18 & 25 & $72,00 \%$ \\
\hline Total & 25 & 24 & 27 & 24 & 100 & $73,00 \%$ \\
\hline PA & $72,00 \%$ & $79,17 \%$ & $66,67 \%$ & $75,00 \%$ & OA & $73 \%$ \\
\hline
\end{tabular}

Table 3 shows the error matrix based on the test area where the best pixel base classification value for the geomorphological zone class is quite high. This shows that the average grade and standard deviation for most objects are classified with high membership values. As we can see from Table 3, the overall accuracy of the pixel base analysis approach is $73 \%$, based on the accuracy of the producers and users of individual classes. Producer's accuracy for " reef flat " class information is $72 \%$, this shows that 72 percent of the reef average was correctly identified, while user accuracy is also $72 \%$, this also shows that $72 \%$ of the area classified as "reef flats" falls into this category. Producer's accuracy for the "reef crest" class information was $79,17 \%$, this shows that $79,17 \%$ of the "reef crest " was correctly identified while user accuracy was $76 \%$, this result showed that $76 \%$ of the area classified as "reef crest" was correct right in this category. Producer's accuracy for " reef slope " class information is $66.7 \%$, this shows that 66.7 $\%$ of the reef average was correctly identified, while user accuracy is $72 \%$, this shows that $72 \%$ of the area classified as "reef slope" falls into this category.

Object Based Classification: Processing and addressing of a certain scale is the task of each object-based classification. Image information is represented by the difference in scale $h$ based on the average size of the object image and the same imagery can be segmented into smaller or larger objects [14]. Object-based classification does not depend on the value of individual pixels, but the value of pixels that have a degree of similarity in groups and neighbors. The next process is the segmentation process where groups of pixels that have the same characteristics are identified. The process of segmentation depends on the threshold of similarity and spectral similarity which then results in the number of variables and the size of the object [15]. In addition, the objects are described by shape, size, tone, texture, compactness, and other characteristics describing the spatial features of the object [16]. Geomorphological zones formed by the classification assignment process of the differences of all these variables. In object-based classification, the term inheritance is known where the characteristics of sub-objects inherit the characteristics of super objects in the upper hierarchy [11].

As a result of classification, seven geomorphology zones were distinguished including land, deep sea, reef crest, reef slope, reef flat and lagoon (Figure 2). The output of the image is a thematic map that accurately results in the classification process without errors or bias [11]. There are a number of equations that can show the level of error statistically, such as producer accuracy, user accuracy, overall accuracy and Kappa which can be calculated using the confusion matrix [17].

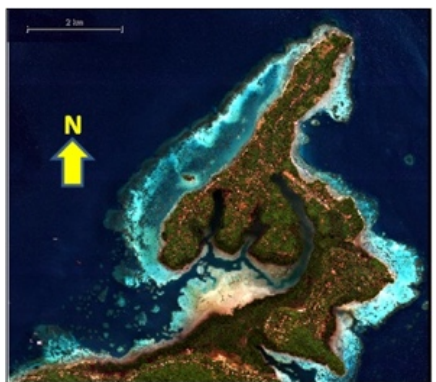

Figure 1 Research Site map

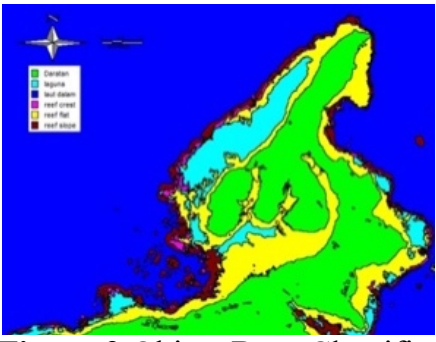

Figure 2 Object Base Classification Result

Standard confusion matrices are used to assess accuracy based on field data and are then classified into five geomorphological zones. As we can see in table 4, the producer accuracy, user accuracy, and overall accuracy for three classification methods.

Table 4 Accuracy assessment results Object Base Classification

\begin{tabular}{|ccccccc|}
\hline Image $\backslash$ Survey & Reef Flat & Reef Crest & Reef Slope & Lagoon & Total & UA \\
\hline Reef Flat & 28 & 1 & 2 & 1 & 32 & $87.50 \%$ \\
\hline Reef Crest & & 28 & 2 & & 30 & $93.33 \%$ \\
\hline Reef Slope & 1 & 2 & 27 & 2 & 32 & $84.38 \%$ \\
\hline Lagoon & 2 & & 1 & 26 & 29 & $89.66 \%$ \\
\hline Total & 31 & 31 & 32 & 29 & 123 & \\
\hline PA & $90.32 \%$ & $90.32 \%$ & $84.38 \%$ & $89.66 \%$ & OA & $88.62 \%$ \\
\hline
\end{tabular}


Riegl, H. Yamano, W.H. White, M. Zubia, J.C.Brock, S.R. Phinn, A. Naseer, B.G. Hatcher, and F.E. MullerKarger. (2004) Multi-site evaluation of IKONOS data for classification of tropical coral reef environments. Remote Sensing of Environment, 88(1-2): p128-143.

[3] Maeder J, Narumalani S, Rundquist DC, Perk RL, Schalles J, Hutchins K, Keck J (2002) Classifying and mapping general coral-reef structure using Ikonos data. Photogr Eng Remote Sens 68:1297-1305

[4] Mumby, P.J. and A.J. Edwards. (2004) Mapping marine environments with IKONOS imagery: enhanced spatial resolution can deliver greater thematic accuracy. Remote Sensing of Environment, 82(2-3): p248-257.

[5] Robinson, J.A., Feldman, G.C., Kuring, N., Franz, B., Green, E., Noordeloos, M., Stumpf, R.P., 2000.

Data fusion in coral reef mapping: working at multiple scales with SeaWiFS and astronaut photography, Proceedings of the 6th International Conference on Remote Sensing for Marine and Coastal Environments, Charleston, South Carolina, pp. 473-483.

[6] Stumpf, R. P., and J. R. Pennock. 1989. Calibration of a general optical equation for remote sensing of suspended sediments in a moderately turbid estuary, J. Geophys. Res. Oceans 94: 14,363-14,371.

[7] Barlow, J., Franklin, S., and Martin, Y. "High spatial resolution satellite imagery, DEM derivatives, and image segmentation for the detection of mass wasting processes", Photogramm. Eng. Rem. S., 72(6), 687-692, 2006. Web. Nov. 2012.

[8] Chang, Kuan-Tsung, Jun-King Liu, and Chu-I Wang. "An Object-oriented Analysis for Characterizing the Rainfall-induced Shallow Landslide." Journal of Marine Science and Technology 20.6 (2012): 647-56. Web. Dec. 2012

This material is based upon work partially supported by LAPAN. Special thanks to my committee: Gatot Winarso, Remote Sensing Application Centre department, LAPAN, Jakarta. To Dr. Sukris Sutiyatno as Director of STMIK Bina Patria which aided greatly in the completion of this research.

\section{REFERENCES}

[1] Wilkinson, C., ed. (2008) Status of Coral Reefs of the World: 2008, 304. Townsville: Global Coral Reef Monitoring Network and Reef and Rainforest Research Centre.

[2] Andréfouët, S., PKramer, D. Torres-Pulliza, K.E. Joyce, E.J. Hochberg, R.Garza-Perez, P.J. Mumby, B.
[9] Martha, Tapas R. Detection of Landslides by Object Oriented Image Analysis. Diss. University of Twente, 2011. Enschede: ITC, 2011. Print. Oct. 2011

[10] Congalton, R.G. and K. Green. (1999) Assessing the accuracy of remotely sensed data: principles and practices. CRC Press, Inc., Florida. 130p.

[11] Foody, Giles M. "Classification accuracy comparison: hypothesis tests and the use of confidence intervals in evaluations of difference, equivalence and non-inferiority." Remote Sensing of Environment 113.8 (2011): 1658-1663.

[12] Digital Globe Inc. Radiometric Use of WorldView-2 Imagery [Internet]. 2010. 
Northern Germany And Wye Downs," UK. J. for Nature Conservation, 13(2-3), 75-89, (2005).

Barrier Reef." Elsevier, Amsterdam.

[14] Walsh, S. J. et al., "Quickbird And Hyperion Data Analysis of An Invasive Plant Species In The

Galapagos Islands of Ecuador: Implications For Control And Land Use Management," Remote Sensing of Environment, 112(5), 1927-1941, (2008).

[15] Kok, R., Schneider, T. and Ammer, U. “Object Based Classification And Applications In The Alpine Forest Environment," International Archives of

Photogrammetry and Remote Sensing, (1999).

[16] Bock, M. et al., "Object-Oriented Methods For Habitat Mapping At Multiple Scales-Case Studies From
[17] Su, W. Zhang, C., Zhu, X. and Daoliang, L.“A Hierarchical Object Oriented Method For Land Cover Classification Of SPOT 5 Imagery," WSEAS Trans Inform Sci Appl.6(3):437-446, (2009).

[18] Gao,Y., Mas, J.F., Maathuis, B.H.P., Xiangmin, Z. and Van Dijk, P. M. "Comparison of Pixel-Based And ObjectOriented Image Classification ApproachesA Case Study In A Coal Fire Area, Mongolia, China," Int. J. of Remote Sens, 27, 4039-4051, (2006). 